\title{
Envelhecimento acelerado em sementes de trigo
}

\author{
Accelerated aging in wheat seeds
}

\author{
Daniele Cardoso Pedroso $^{\mathrm{I}^{*}}$ Lilian Madruga Tunes $^{\mathrm{I}}$ Ana Paula Barbieri ${ }^{\mathrm{II}}$ \\ Antonio Carlos Souza Albuquerque Barros ${ }^{\text {III }}$ Marlove Fátima Brião Muniz ${ }^{I}$ \\ Vanessa Ocom Menezes ${ }^{I}$
}

\begin{abstract}
O presente trabalho teve o objetivo de estudar RESUMO procedimentos de condução do teste de envelhecimento acelerado para determinar o potencial fisiológico de sementes de trigo. Utilizaram-se quatro cultivares: 'Pampeano', 'Safira', 'Ônix' e 'Jaspe'. Foram realizadas determinações de teor de água e avaliações de germinação, emergência, indice de velocidade de emergência e teste de envelhecimento acelerado. $O$ teste de envelhecimento acelerado foi conduzido de três formas: teste tradicional (água), teste com solução saturada de $\mathrm{NaCl}$ (11g de $\mathrm{NaCl}$ por $100 \mathrm{~mL}$ de água) e teste com solução supersaturada de $\mathrm{NaCl}$ (40g de $\mathrm{NaCl}$ por $100 \mathrm{~mL}$ de água), nos periodos de 48, 72 e $96 \mathrm{~h}$ de permanência na câmara de envelhecimento a $42^{\circ} \mathrm{C}$. O teste de envelhecimento acelerado com solução saturada de $\mathrm{NaCl}$, no periodo de 96h, foi o mais adequado para avaliar o potencial fisiológico das sementes de trigo, em alto, médio e baixo vigor.
\end{abstract}

Palavras-chave: solução salina, germinação, vigor, Triticum aestivum.

\section{ABSTRACT}

This research aimed to study procedures for conducting the accelerated aging test to determine the physiological potential of wheat seeds. The experiment was conducted with four varieties: 'Pampeano', 'Sapphire', Onyx' and 'Jasper'. Tests were performed with water content, germination, seedling emergence, speed of emergence and accelerated aging. The accelerated aging test was conducted in three stages: traditional (water), test using saturated $\mathrm{NaCl}$ solution (11g of $\mathrm{NaCl}$ per $100 \mathrm{~mL}$ of water) and with the use of supersaturated solution of $\mathrm{NaCl}(40 \mathrm{~g}$ of $\mathrm{NaCl}$ per $100 \mathrm{~mL}$ of water), each for 48,72 and $96 \mathrm{~h}$ at $42^{\circ} \mathrm{C}$. The accelerated aging test with saturated solution of $\mathrm{NaCl}$, within $96 \mathrm{~h}$ was suitable to evaluate the physiological potential of seeds of wheat in high, medium and low vigor.

Key words: solution saline, germination, vigor, Triticum aestivum.

O teste de envelhecimento acelerado consiste em avaliar a resposta das sementes, por meio do teste de germinação, após terem sido submetidas a condições de estresse: temperatura elevada e umidade relativa próxima a $100 \%$, por determinado tempo (MARCOS FILHO, 1999). A temperatura e o período de permanência das sementes na câmara de envelhecimento variam conforme a espécie. Para o trigo, têm sido indicadas as combinações de $42^{\circ} \mathrm{C}$ por $24 \mathrm{e}$ $48 \mathrm{~h}$ (MAIA et al., 2007) ou $43^{\circ} \mathrm{C}$ por $48 \mathrm{~h}$ (LIMA et al., 2006).

A absorção de água pelas sementes é um fator que pode interferir na interpretação dos dados do teste de envelhecimento acelerado (MARCOS FILHO, 2005). Por esse motivo, vêm sendo estudadas alternativas para a condução do envelhecimento acelerado, como a substituição da água por soluções salinas, a fim de controlar o processo de embebição e, assim, a deterioração das sementes (JIANHUA \& McDONALD, 1996), sem reduzir a sensibilidade do teste.

\footnotetext{
'Departamento de Defesa Fitossanitária, Universidade Federal de Santa Maria (UFSM). Avenida Roraima, 1000, 97105-900, Santa Maria, RS, Brasil. E-mail: danibioufsm@yahoo.com.br. *Autor para correspondência.

"Departamento de Fitotecnia, UFSM, Santa Maria, RS, Brasil.

II'Universidade Federal de Pelotas (UFpel), Rua Gomes Carneiro, 1, 96001-970, Pelotas, RS, Brasil.
} 
Diante do exposto, o presente trabalho teve por objetivo estudar procedimentos para a condução do teste de envelhecimento acelerado para determinar o potencial fisiológico de sementes de trigo (Triticum aestivum L.) das cultivares 'Pampeano', 'Safira', 'Ônix' e 'Jaspe'.

Para avaliar o desempenho das sementes dessas quatro cultivares quanto ao potencial fisiológico, conduziram-se as seguintes determinações: teor de água, germinação, emergência, índice de velocidade de emergência (IVE) e envelhecimento acelerado. $\mathrm{O}$ teor de água e a germinação foram conduzidos de acordo com as Regras de Análises de Sementes-RAS (BRASIL, 2009). Quanto à emergência, foram avaliadas quatro repetições de 50 sementes para cada cultivar, semeadas em bandejas de isopor com células separadas. As contagens foram realizadas diariamente, às 18 horas, iniciadas a partir do terceiro dia e finalizadas no décimo segundo dia após a semeadura, sendo o critério para avaliação o de plântulas com o coleóptilo acima do solo, conforme RITCHIE et al. (1993). O índice de velocidade de emergência (IVE) foi realizado conjuntamente com o teste de emergência, através de contagens diárias, no mesmo horário, do número de plântulas emergidas dos três aos doze dias após a semeadura, sendo o critério para avaliação também o de plântulas com o coleóptilo acima do solo, cujo índice foi calculado de acordo com a fórmula proposta por Maguire (1962). O envelhecimento acelerado foi conduzido em caixas plásticas tipo gerbox, contendo $40 \mathrm{~mL}$ de água destilada (tradicional), solução de $\mathrm{NaCl}$ a $76 \%$ (solução supersaturada) e a $94 \%$ (solução saturada), usando-se 40 e $11 \mathrm{~g}$ de $\mathrm{NaCl}$ por $100 \mathrm{~mL}$, respectivamente. As sementes $(30 \mathrm{~g})$ foram dispostas, de maneira uniforme, sobre uma tela de alumínio, o que é tradicional para esse teste. As caixas foram mantidas em estufa do tipo BOD, a $42^{\circ} \mathrm{C}$, durante 48,72 e $96 \mathrm{~h}$. Decorridos os períodos, quatro subamostras de 50 sementes por cultivar foram submetidas ao teste de germinação, seguindo metodologia descrita anteriormente. Paralelamente, foi determinado o teor de água das sementes após cada período de envelhecimento, para verificar a uniformidade das condições do teste, conforme Marcos Filho (1999). Os resultados foram submetidos à análise de variância e as médias comparadas pelo teste de Tukey $(\alpha=0,05)$, empregandose o programa de análises estatísticas Sisvar (FERREIRA, 2000).

Na tabela 1, encontram-se os dados obtidos para o teor de água das sementes, os quais são semelhantes para as sementes das quatro cultivares,
Tabela 1 - Determinações iniciais (teor de água - TA, germinação - G, emergência de plântulas - EP e índice de velocidade de emergência - IVE) de lotes de sementes de trigo das cultivares 'Pampeano', 'Safira', 'Ônix' e 'Jaspe'.

\begin{tabular}{|c|c|c|c|c|}
\hline Cultivar & TA & G & EP & IVE \\
\hline & \multicolumn{4}{|c|}{---------------------------\%\%-------------------------- } \\
\hline 'Pampeano' & 12,08 & $91 \mathrm{a}^{*}$ & $90 a$ & $12,64 \mathrm{a}$ \\
\hline 'Safira' & 12,39 & $90 \mathrm{a}$ & $90 \mathrm{a}$ & $12,49 \mathrm{a}$ \\
\hline 'Ônix’ & 12,45 & $90 \mathrm{a}$ & $92 \mathrm{a}$ & $11,80 \mathrm{a}$ \\
\hline 'Jaspe' & 12,22 & $90 \mathrm{a}$ & $90 \mathrm{a}$ & $12,67 \mathrm{a}$ \\
\hline $\mathrm{CV}(\%)$ & & 1,84 & 3,17 & 4,02 \\
\hline
\end{tabular}

*Médias seguidas das mesmas letras, nas colunas, não diferem entre si pelo teste de Tukey a $5 \%$ de probabilidade.

com variação de até 0,37 pontos percentuais, variação essa inferior à amplitude máxima aceitável, que é de $1 \mathrm{a}$ 2 pontos percentuais (MARCOS FILHO, 1999). Ainda pelos dados da tabela 1, observa-se que as sementes das cultivares não diferiram significativamente quanto ao potencial fisiológico nos testes de germinação, emergência e índice de velocidade de emergência.

$\mathrm{Na}$ figura 1, observa-se o teor de água das sementes atingido após a realização do teste de envelhecimento acelerado tradicional (TR), com o uso de solução salina saturada (SS) e supersaturada de $\mathrm{NaCl}$ (SSS), nos três períodos de exposição. Para o teste de envelhecimento tradicional ( $100 \%$ de umidade relativa) as sementes, das quatro cultivares de trigo, apresentaram os menores teores de água no período de exposição de $48 \mathrm{~h}$, e o mesmo foi aumentando gradativamente com o aumento do tempo de exposição.

As sementes submetidas ao teste de envelhecimento acelerado com solução salina saturada ( $94 \%$ de umidade relativa), no período de 48 e $96 \mathrm{~h}$, ainda tinham alto teor de água, variando de 27 a $21 \%$ de umidade, valores considerados bastante altos. Portanto, o tempo de exposição de $72 \mathrm{~h}$ foi o mais adequado para o teste de envelhecimento com solução salina, no qual foram verificados menores teores de água (Figura 1).

Os resultados demonstraram que o uso de solução supersaturada de $\mathrm{NaCl}$ ( $76 \%$ de umidade relativa) promoveu a redução da velocidade de absorção de água pelas sementes de trigo durante os períodos de envelhecimento (Figura 1), de modo que, com $96 \mathrm{~h}$, as sementes atingiram grau de umidade inferior aos verificados com o uso do protocolo tradicional. Nesse caso, houve variações mais acentuadas no teor de água das sementes ao final do envelhecimento acelerado, confirmando as observações de JIANHUA \& McDONALD (1996), os quais descreveram a 


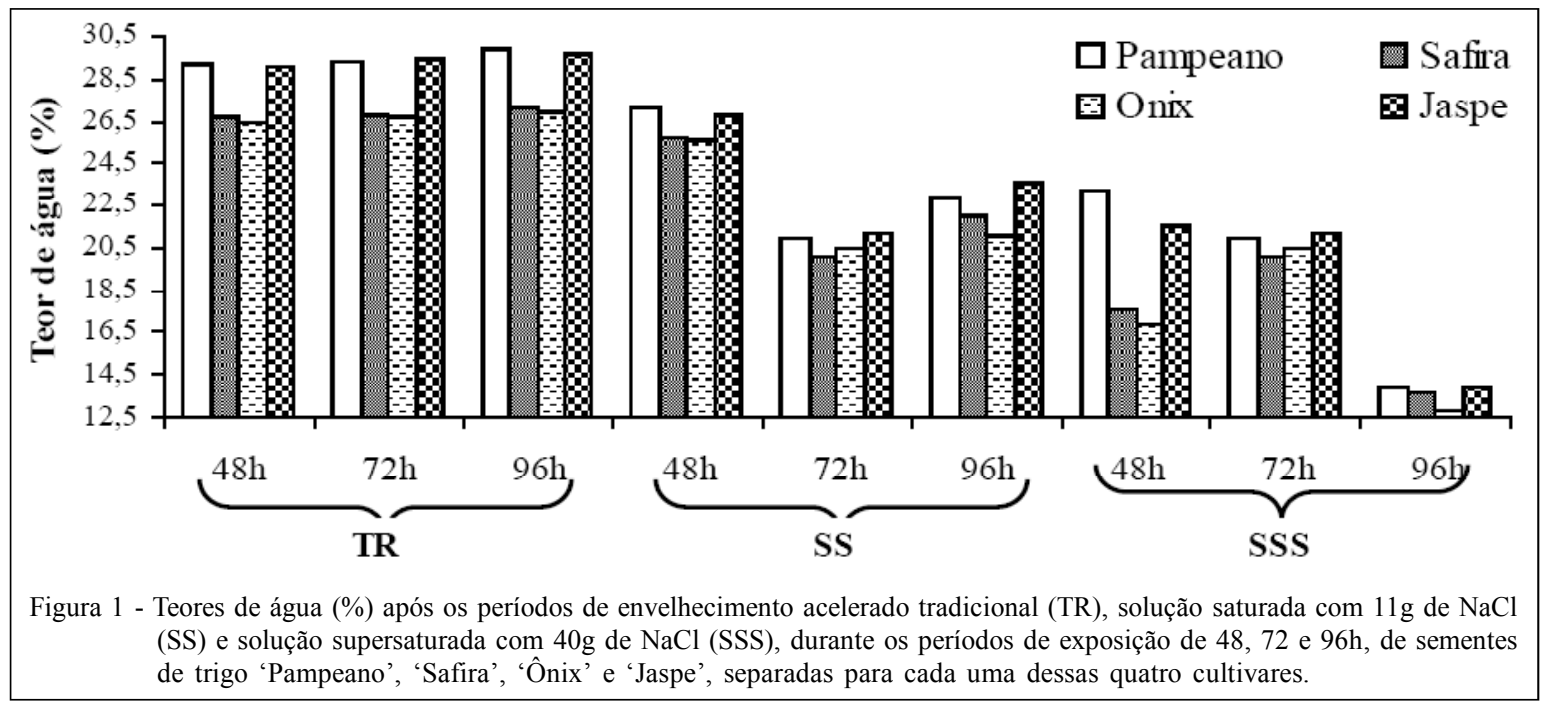

metodologia. No entanto, não há registros do uso dessa metodologia alternativa em sementes de trigo.

Na tabela 2, encontram-se os resultados da germinação obtidos após o teste de envelhecimento acelerado tradicional, envelhecimento com solução saturada e com solução supersaturada de $\mathrm{NaCl}$. No procedimento tradicional, pode-se verificar que, no período de $48 \mathrm{~h}$ de exposição, já ocorreu uma diferenciação entre as sementes das cultivares em função do potencial fisiológico. O melhor desempenho foi obtido com sementes das cultivares 'Pampeano', 'Ônix’ e 'Jaspe', permitindo, assim, classificá-las como as de maior vigor.

Os resultados obtidos no teste de envelhecimento acelerado, conduzido de forma tradicional e com solução saturada no período de exposição de 96h, separam as cultivares em maior número de níveis de vigor. No entanto, ao se analisar os valores de germinação, após o envelhecimento tradicional, notou-se elevada redução na porcentagem de plântulas, indicando que o procedimento que utiliza 96h de exposição (envelhecimento tradicional) não é o mais adequado para o trigo. RAMOS et al. (2004) e TUNES et al. (2009) constataram, também, que o estresse provocado pelo teste de envelhecimento acelerado tradicional $\mathrm{a} 42^{\circ} \mathrm{C}$, por $96 \mathrm{~h}$, foi suficiente para ocasionar uma redução expressiva da germinação de sementes de cenoura e cevada, respectivamente.

Com relação à condução do teste de envelhecimento acelerado com o uso de solução salina (SS) (Tabela 2), observa-se que o período de $48 \mathrm{~h}$ de exposição, para as sementes das quatro cultivares, forneceu informações compatíveis com aquelas dos testes de germinação e emergência de plântulas realizados inicialmente, onde não houve diferenças significativas em relação ao percentual de germinação. Por outro lado, os resultados referentes ao teste de envelhecimento acelerado com solução salina (SS),

Tabela 2 - Porcentagem de germinação (\%) após os períodos de envelhecimento acelerado tradicional (TR), solução saturada com $11 \mathrm{~g}$ de $\mathrm{NaCl}$ (SS) e solução supersaturada com $40 \mathrm{~g}$ de $\mathrm{NaCl}$ (SSS), durante o período de exposição de 48, 72 e 96h, de sementes de trigo 'Pampeano', 'Safira', 'Ônix' e 'Jaspe'.

\begin{tabular}{|c|c|c|c|c|c|c|c|c|c|}
\hline & \multicolumn{3}{|c|}{-----------Tradicional----------- } & \multicolumn{3}{|c|}{ 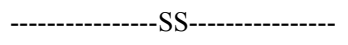 } & \multicolumn{3}{|c|}{-----------------SSS----------------- } \\
\hline & $48 \mathrm{~h}$ & $72 \mathrm{~h}$ & $96 \mathrm{~h}$ & $48 \mathrm{~h}$ & $72 \mathrm{~h}$ & $96 \mathrm{~h}$ & $48 \mathrm{~h}$ & $72 \mathrm{~h}$ & $96 \mathrm{~h}$ \\
\hline Cultivar & \multicolumn{9}{|c|}{$\%$} \\
\hline 'Pampeano' & $88 \mathrm{a}$ & $70 \mathrm{~b}$ & $53 \mathrm{a}$ & $95 \mathrm{a}$ & $91 b$ & $77 \mathrm{~b}$ & $95 \mathrm{a}$ & $94 \mathrm{a}$ & $90 \mathrm{~b}$ \\
\hline 'Safira' & $77 \mathrm{~b}$ & $75 b$ & $21 \mathrm{c}$ & $96 a$ & $92 \mathrm{ab}$ & $68 \mathrm{c}$ & $96 a$ & $97 \mathrm{a}$ & $93 a$ \\
\hline ‘Ônix’ & $91 \mathrm{a}$ & $79 a$ & $29 b$ & $97 \mathrm{a}$ & $95 \mathrm{a}$ & $84 a$ & $98 \mathrm{a}$ & $95 \mathrm{a}$ & $95 \mathrm{a}$ \\
\hline 'Jaspe' & $86 a$ & $76 b$ & $42 \mathrm{a}$ & $91 \mathrm{a}$ & $90 \mathrm{~b}$ & $78 \mathrm{a}$ & $94 \mathrm{a}$ & $96 a$ & $90 \mathrm{~b}$ \\
\hline $\mathrm{CV}(\%)$ & \multicolumn{6}{|c|}{ 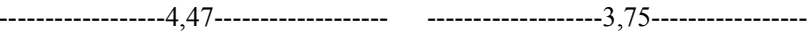 } & .---.-. & $-2,24$ & ..-. \\
\hline
\end{tabular}

"Médias seguidas das mesmas letras nas colunas, para cada cultivar e tempo de exposição, não diferem entre si pelo teste de Tukey a $5 \%$ de probabilidade. $\mathrm{CV}=$ coeficiente de variação. 
para o período de $72 \mathrm{~h}$ de exposição das sementes, proporcionaram a separação das sementes das cultivares em níveis de vigor sem prejudicar o potencial germinativo das mesmas, indicando 'Safira' e 'Ônix' como de qualidade superior e 'Pampeano' e 'Jaspe' como as de qualidade inferior.

Com base nos resultados da tabela 2 , podese afirmar que a solução salina de $\mathrm{NaCl}$ (SS) com um maior período de exposição afeta, de forma prejudicial, o processo germinativo de sementes de trigo. Assim sendo, seria esperado que as condições de envelhecimento, com uso de solução saturada de $\mathrm{NaCl}$, promovessem efeitos menos severos nas sementes em decorrência dos menores teores de água atingidos durante o envelhecimento.

Mediante os resultados obtidos para o teste de envelhecimento com solução supersaturada de $\mathrm{NaCl}$ (SSS) (Tabela 2), pode-se observar que ocorreram diferenças significativas entre as cultivares apenas no período de $96 \mathrm{~h}$. As sementes mais vigorosas foram encontradas nas cultivares 'Safira' e 'Ônix', sendo esses resultados similares aos encontrados no teste com solução salina saturada no período de exposição de $72 \mathrm{~h}$.

Observa-se também que o teste de envelhecimento acelerado com solução de $\mathrm{NaCl}$ nas duas concentrações testadas é promissor para utilização em programas de controle de qualidade, pois, além de proporcionar condições para absorção de menores quantidades de água de maneira mais uniforme pelas sementes, requer equipamentos e metodologia semelhantes ao método tradicional.

O teste de envelhecimento acelerado com solução saturada de $\mathrm{NaCl}$, no período de $96 \mathrm{~h}$, é o mais adequado para avaliar o potencial fisiológico das sementes de trigo, em alto, médio e baixo vigor.

\section{REFERÊNCIAS}

BRASIL. Ministério da Agricultura, Pecuária e Abastecimento. Regras para análise de sementes. Brasília, 2009. 399p.

FERREIRA, D.F. Análises estatísticas por meio do SISVAR para windows versão 4.0. In: REUNIÃO ANUAL DA REGIÃO BRASILEIRA DA SOCIEDADE INTERNACIONAL DE BIOMETRIA, 45., 2000, São Carlos. Anais... São Carlos: UFSCAR, 2000. p.225-258.

JIANHUA, Z.; McDONALD, M.B. The saturated salt accelerated aging test for small-seeded crops. Seed Science and Technology, v.25, n.1, p.123-131, 1996.

LIMA, T.C. et al. Avaliação do potencial fisiológico de sementes de trigo (Triticum aestivum L.) pelo teste de envelhecimento acelerado. Revista Brasileira de Sementes, v.57, n.2, p.106$113,2006$.

MAGUIRE, J.D. Speed of germination aid in selection and evaluation for seeding emergence and vigor. Crop Science, v.2, n.2, p.176-177, 1962.

MAIA, A.R. et al. Teste do envelhecimento acelerado na avaliação da qualidade fisiológica de sementes de trigo. Ciência e Agrotecnologia, v.31, n.3, p.678-684, 2007. Disponível em: $<$ http://www.scielo.br/pdf/cagro/v31n3/a12v31n3.pdf $>$. Acesso em: 10 abr. 2010. doi: 1590/S1413-70542007000300012.

MARCOS FILHO, J. Testes de vigor: importância e utilização. In: KRZYZANOWSKI, F.C. et al. Vigor de sementes: conceitos e testes. Londrina: ABRATES, 1999. p.1.1-1.21.

MARCOS FILHO, J. Fisiologia de sementes de plantas cultivadas. Piracicaba: FEALQ, 2005. 495p.

RAMOS, N.P. et al. Envelhecimento acelerado em sementes de rúcula (Eruca sativa L.). Revista Brasileira de Sementes, v.26, n.1, p.98-103, 2004.

RITCHIE, S.W. et al. How a corn plant develops. Ames: Iowa State University, 1993. 26p.

TUNES, L.M. et al. Teste de envelhecimento acelerado em cevada. Magistra, v.21, n.2, p.111-119, 2009. 